\title{
Local expression of IDO, either alone or in combination with CD40Ig, IL10 or CTLA4Ig, inhibits indirect xenorejection responses
}

Mulley WR, Li YQ, Wee JL, Dodge N, Christiansen D, Simeonovic C, Ierino FL, Sandrin MS. Local expression of IDO, either alone or in combination with CD40Ig, IL10 or CTLA4Ig, inhibits indirect xenorejection responses.

Xenotransplantation 2008; 15: 174-183. (c) 2008 Blackwell Munksgaard

Abstract: Background: To overcome cell-mediated xenorejection by transgenic expression of immunomodulatory molecules by a graft, it is likely that expression of multiple molecules will be required. Previous studies support the use of the immunomodulatory agents indoleamine 2,3-dioxygenase (IDO), CD40Ig, interleukin 10 (IL10), and CTLA4Ig for suppression of rejection responses. We examined the effects of local expression of these molecules by a porcine cell line (PIEC) on indirect murine xenorejection responses in vitro and in vivo.

Methods: The PIEC stable lines expressing IDO, CD40Ig, and IL10 as single molecules were generated. In addition, PIEC lines expressing IDO with either CD40Ig, IL10 or CTLA4Ig were generated to produce cell lines expressing two molecules. BALB/c mice were primed with wild type PIEC, followed by harvesting splenocytes used as responder cells and PIEC expressing immunomodulatory molecules as stimulators, in proliferation and cytokine assays. In vivo effects of modified PIEC were examined by transplantation of PIEC lines expressing the immunomodulatory molecules under the renal capsule of naïve mice. PIEC grafts were harvested for histological evaluation at days 7 and 14 . Results: Proliferation of primed BALB/c splenocytes was inhibited most significantly by IDO compared with control cells $(49 \%, \mathrm{P}=0.02)$. In addition both Th1 (interferon-gamma) and Th2 (IL4 and IL10) cytokines were markedly inhibited in vitro by IDO expression. IL10 expressing cells did not inhibit proliferation as potently $(37 \%, \mathrm{P}=0.03)$ whilst CD40Ig lead to an increase in proliferative responses $(59 \%$, $\mathrm{P}=0.02$ ). Co-expression of CD40Ig, IL10, and CTLA4Ig with IDO resulted in further modest reductions in proliferation compared with IDO expression alone. When transplanted under the renal capsule of BALB/c mice, those grafts expressing IDO demonstrated significantly lower levels of lymphocyte infiltration at days 7 and 14 than control grafts and those expressing CD40Ig, CTLA4Ig or IL10 alone. Grafts co-expressing IDO and a second molecule were no better protected than those expressing IDO alone. Graft cell viability (PIECs) was reduced in some IDO expressing grafts suggesting high levels of IDO expression may inhibit PIEC viability, however, grafts co-expressing IDOCTLA4Ig and IDO-IL10 were not affected in this way.

Conclusion: Indoleamine 2,3-dioxygenase appears to be a potent molecule for protecting xenografts from cell-mediated rejection responses activated via the indirect pathway. Co-expression of IDO with both CTLA4Ig and IL10 warrants further investigation. Overall these findings support pursuing further studies, in larger animal models, to determine whether increased IDO activity within the graft itself can attenuate xenorejection responses.

\section{William R. Mulley, ${ }^{1}$ Yu Oin Li, ${ }^{1}$ Janet L. Wee, ${ }^{1}$ Natalie Dodge, ${ }^{1}$ Dale Christiansen, ${ }^{1}$ Charmaine Simeonovic, ${ }^{2}$ Francesco L. lerino $^{1,3}$ and Mauro S. Sandrin ${ }^{1}$}

${ }^{1}$ Department of Surgery, Austin Health and University of Melbourne, Melbourne, Vic., ${ }^{2}$ Division of Immunology and Genetics, The John Curtin School of Medical Research, The Australian National University, Canberra, ACT, ${ }^{3}$ Department of Nephrology, Austin Health, Melbourne, Vic., Australia

Key words: graft rejection - lentivirus transplantation tolerance

Abbreviations: IDO, indoleamine 2,3-dioxygenase; CTLA4lg, cytotxic T-lymphocyte antigen 4-immunoglobulin; IL, interleukin; CD40lg, CD40-immunoglobulin; IFN, interferon; PIEC, porcine iliac artery endothelial cell; Th, T-helper; Treg, T-regulatory cell; LacZ, beta galactosidase; GFP, green fluorescent protein.

Address reprints request to Professor Mauro Sandrin, Department of Surgery, Austin Health and University of Melbourne, Melbourne, Vic. 3084, Australia (E-mail: m.sandrin@ari.unimelb.edu.au) 


\section{Background}

Indirect antigen presentation is an important route to graft loss in xenotransplantation [1]. The indirect pathway is also relevant in allorejection particularly for grafts which express little major histocompatibility complex (MHC) class II such as islet cell clusters [2]. MHC class II on donor cells is required for direct antigen presentation to recipient $\mathrm{CD} 4^{+} \mathrm{T}$ cells, whilst indirect presentation results from antigen presentation of graft peptides by recipient antigen-presenting cells to recipient $T$ cells [3]. Overcoming indirect xenorejection pathways by local immunomodulatory molecule expression by the graft itself would reduce or eliminate the need for systemic immunosuppressive medications and their associated toxicities, providing impetus for the use of transgenic porcine islet grafts in humans. Our group has previously reported on the in vitro effects of CTLA4Ig in this setting [4], however, the effects of indoleamine 2,3-dioxygenase (IDO), CD40-Immunoglobulin (CD40Ig) and interleukin-10 (IL10) local overexpression by cellular grafts on indirect xenoresponses have not previously been reported.

Indoleamine 2,3-dioxygenase is an intracellular enzyme which catabolizes tryptophan resulting in tryptophan depletion and increased levels of tryptophan metabolites (kynurenines) [5,6]. T cells exposed to reduced tryptophan and increased kynurenine concentrations are prone to apoptosis and in some conditions can develop T-regulatory features [5,6]. IDO has thus become an important molecule for study in transplantation. IDO overexpression in rodent cornea, lung and pancreas allografts has resulted in prolonged survival [7-9]. Currently there are few reported studies examining IDO overexpression in transplantation overall and fewer examining the effect of IDO on sensitized recipients or in xenografts. Alexander et al. [7] transduced islet cells with IDO cDNA, using an adenoviral vector, prior to transplant into NODSCID mice. When reconstituted with diabetogenic splenocytes, islet graft survival was modestly increased with a reduction in infiltrating cells in the IDO expressing grafts. In a skin xenograft model, fibroblasts transfected with IDO cDNA exhibited prolonged survival and reduced $\mathrm{CD}^{+}$ cellular infiltration at days 8 and 28 [10].

Blocking CD40:CD40L costimulation of $\mathrm{T}$ cells has been shown to prolong allograft and xenograft survival in a variety of rodent models and allograft survival in primates $[11,12]$. Blockade with a monoclonal antibody has been used in human trials but ongoing studies were precluded because of an increased incidence of thrombotic events due to CD40L expression on platelets. Therefore, efforts have been redirected to developing CD40 blocking antibodies [12]. CD40Ig remains a useful molecule for studying disruption of this pathway in animal studies.

Interleukin 10 is a pleiotropic cytokine with mixed outcomes as an immunomodulatory molecule in transplant studies. Transplant outcomes with systemic IL10 therapy may be summarized as: (i) IL10 administered prior to grafting can prolong graft survival; (ii) IL10 administered at the time of, or after grafting does not inhibit and may accelerate graft rejection; (iii) viral IL10 is more consistently effective in prolonging graft survival than cellular IL10; and (iv) high dose IL10 may accelerate rejection compared with the protective effects of low dose IL10 [13,14].

CTLA4Ig therapy has been effective at prolonging graft survival in a variety of experimental models, including in humans, as we and others have described previously $[4,15]$.

To overcome cellular xenorejection responses it appears likely that a combination of immunomodulatory molecules will be required as no single agent has yet proven to be sufficient. The effects of IDO coexpression with other molecules have not previously been reported. To address these two areas, we studied the effects of local IDO, IL10, and CD40Ig expression by a class II-deficient porcine endothelial cell line on indirect xenorejection responses in vitro and in vivo. In addition co-expression of IDO with a second immunomodulatory molecule (CTLA4Ig, CD40Ig or IL10) was examined. IDO proved to be a potent inhibitor of lymphocyte proliferation and cytokine production in vitro and inhibited lymphocyte infiltration of grafts in vivo. CD40Ig and IL10 expressing cells were less effective, whilst the combinations of IDO-IL10 and IDO-CTLA4Ig appear to hold some promise.

\section{Materials and methods}

Generation and maintenance of stable IDO expressing porcine endothelial cells

The cDNA for murine CTLA4, CD40, IL10, human IDO and the immunoglobulin ([Ig] hinge, $\mathrm{CH} 2$, and $\mathrm{CH} 3$ regions) were isolated by PCR from gene libraries. Lentivirus was produced using the ViraPower ${ }^{\mathrm{TM}}$ lentiviral expression system (Invitrogen Corporation, Carlsbad, CA, USA). The cDNA encoding the genes of interest were cloned into the entry vector pENTR11 (Invitrogen) before recombination into the lentiviral vector pLenti6/ V5-DEST (Invitrogen). Lentivirus for CTLA4Ig, CD40Ig, and IL10 was produced and stored as 
previously described; using 293FT packaging cells [4]. Single expressing pig iliac artery endothelial cell (PIEC) lines were generated for CD40Ig, CTLA4Ig, IL10 and the control molecules beta galactosidase (LacZ) and green fluorescent protein (GFP) as previously described [4]. A stably transfected PIEC line producing IDO conjugated to a Flag tag (Sigma, St Louis, MO, USA) was generated by lipofection (in the expression vector pcDNA3 [Invitrogen]), neomycin selection and cloning by limiting dilution. The PIEC line, was a kind gift from Dr. K. Welsh (Oxford, UK). PIECs do not express MHC class II after passaging in tissue culture ([16], and our own observations).

To establish co-expression of CD40Ig, CTLA4Ig or IL10 with IDO, PIEC-IDO cells were transduced with lentiviral vectors for CD40Ig, CTLA4Ig and IL10 and stable expressing clones selected after 10 days exposure to both neomycin and blasticidin. Blasticidin resistance is transmitted with the lentiviral vector.

The PIECs were maintained in DME media (JRH Biosciences, Lenexa, KS, USA) supplemented with $20 \mu \mathrm{g} / \mathrm{ml}$ glutamine, $100 \mathrm{IU} / \mathrm{ml}$ penicillin, $100 \mu \mathrm{g} / \mathrm{ml}$ streptomycin, and $10 \%$ foetal calf serum ([FCS], JRH Biosciences, Brooklyn, Vic., Australia) in a humidified $10 \% \mathrm{CO}_{2}$ atmosphere. Neomycin $(500 \mu \mathrm{g} / \mathrm{ml})$ was added to PIEC-IDO cell cultures, blasticidin $(10 \mu \mathrm{g} / \mathrm{ml})$ to single expressing cells generated by lentiviral transduction and both to dual expressing cells to maintain clonality. Proliferation assays were performed in RPMI (JRH Biosciences, Lenexa, KS, USA) with $10 \%$ FCS supplemented with $20 \mu \mathrm{g} / \mathrm{ml}$ glutamine, $100 \mathrm{IU} / \mathrm{ml}$ penicillin, and $100 \mu \mathrm{g} / \mathrm{ml}$ streptomycin.

Assessing transgene expression from stable clones after lentiviral transduction

To detect IDO production, $20 \mu \mathrm{l}$ of PIEC-IDO cell lysate (generated from $1 \times 10^{6}$ cells) was analyzed by Western blot, probed with anti-Flag BioM2 antibody (Sigma). An IDO functional assay was also performed using a previously described method [17].

To detect CTLA4Ig and CD40Ig expression by PIECs, tissue culture supernatant (SN) was recovered from PIECs, originally seeded at $2 \times 10^{5}$, and left in unchanged media for 4 to 7 days. Twenty microliters was run on a Western blot and then probed with an HRP-conjugated sheep-anti-human Ig antibody (Silenus, Melbourne, Vic., Australia).

To detect IL10 secretion an ELISA was performed, SN $(50 \mu \mathrm{l})$ from PIEC-IL10 cells was added to triplicate wells of a plate coated with anti-
muIL10 (Pharmingen, San Jose, CA, USA). To develop the ELISA, a biotinylated anti-muIL10 antibody (Pharmingen) was used and then an HRP-conjugated streptavidin secondary antibody applied. A commercially produced mIL10 $(10 \mu \mathrm{g} /$ $\mathrm{ml}$ [Pharmingen]) was used as a positive control.

Xenogeneic proliferation assays

Gamma-irradiated (90 Gy) PIECs $\left(1 \times 10^{4}\right.$ cells/ well) were used for stimulator cells in sterile 96 -well u-bottom plates. Responder cells, added after $24 \mathrm{~h}$, were splenocytes from BALB/c mice primed in vivo by intraperitoneal injection with wild-type PIECs a minimum of 10 days earlier. Red cells were lysed, after spleen maceration, with $0.84 \% \mathrm{NH}_{4} \mathrm{Cl}$ at $37^{\circ} \mathrm{C}$ for $5 \mathrm{~min}$. Splenocytes were added to the proliferation assays at $2 \times 10^{5}$ cells/well. Assays were incubated at $37^{\circ} \mathrm{C}$ for 3 to 5 days. Cells were cultured in $200 \mu \mathrm{l}$ of media/well. Assays included control wells containing cells only; media only, and anti-self responses. All assays used triplicate wells and were repeated at least three times. Results are from peak proliferation time points (day 3 of culture).

\section{Harvesting and counting}

Tritiated thymidine $\left({ }^{3} \mathrm{H}\right)$ was added to wells $16 \mathrm{~h}$ prior to harvest. Cells were harvested onto glass fiber filters (Packard, Groningen, The Netherlands) using an automated cell harvester (Brandel $\mathrm{CH}$ 055, Gaithersburg, MD, USA). Cell-bound ${ }^{3} \mathrm{H}$ was counted on a Topcount.nxt scintillation counter (Packard, Meriden, CT, USA).

\section{ELISPOT assays}

Enzyme-linked immunospot assays (ELISPOT) were conducted to determine cytokine production by splenocytes in the proliferation assays. ELISPOT plates (Millipore, Billerica, MA, USA) were coated with monoclonal anti-murine antibodies $(5 \mu \mathrm{g} / \mathrm{ml})$ to interferon-gamma (IFN- $\gamma$; Mabtech, Stockholm, Sweden), IL4 or IL10 (Becton Dickinson, San Jose, CA, USA) and blocked with 10\% FCS. Ninety-six well plates were set up in an identical fashion to the direct contact proliferation assays and cultured for $48 \mathrm{~h}$ before transfer to the ELISPOT plates for a further $24 \mathrm{~h}$ of culture. Cells were then removed; plates washed $(0.5 \%$ Tween 20 in PBS) and developed using biotinylated anti-murine monoclonal antibodies (IFN- $\gamma$ [Mabtech] and IL-4 and IL-10 [Becton Dickinson]) and then streptavidin-alkaline phosphatase (Mabtech). Spots were detected using a colorimetric alkaline phosphatase kit (Bio-Rad, 
Hercules, CA, USA) and counted using an ELISPOT reader (AID, Strassberg, Germany) and AID ELISPOT software.

PIEC cellular grafts under the kidney capsule

The PIECs recovered from tissue culture were washed twice in usual culture medium and then embedded in recipient blood clots as previously described [18]. Briefly $2.5 \times 10^{6}$ PIECs/well were pelleted in 96-well u-bottom plates and $\mathrm{SN}$ removed. Ten microliters of recipient type $(\mathrm{BALB} / \mathrm{c})$ blood was added and mixed to embed cells. The blood was allowed to clot for $10 \mathrm{~min}$ before 2 clots per mouse were inserted under the left renal capsule. Kidneys were recovered at day 7 or $14(n=3$ per group per time point) for histology.

\section{Histology}

Grafts were fixed in 4\% formaldehyde, embedded in paraffin, sectioned at $50 \mu \mathrm{m}$ intervals and stained with hematoxylin and eosin. Cell counts were performed on the tissue sections using a modification of a previously described method [19,20]. In brief $40 \times$ magnification digital images of the grafts were taken at pre-determined intervals using a Coolscope microscope (Nikon, Kanagawa, Japan). Three grafts per time interval/cell type were examined $(\mathrm{n}=2$ for PIEC-IDO at day 14) with a minimum of three images counted per graft. Digital images were analyzed using Image-Pro Plus, version 4.5 (Media Cybernetics, Silver Spring, MD, USA). Each image was divided into twelve $54 \times 54 \mu \mathrm{m}$ squares with the middle six squares, referred to hereafter as "a field", assessed. PIECs, lymphocytes, and neutrophils were counted in each section at a distance of 25 to $50 \mu \mathrm{m}$ from the graft/kidney interface.

\section{Statistical analysis}

The Student's $t$-test was used to determine P-values in proliferation, ELISA and ELISPOT assays (Microsoft Excel). Means and standard errors of the mean of the total number of each cell type per field were calculated using GraphPad Prism version 4.00 (San Diego, CA, USA) for analysis of histology data.

\section{Results}

Expression of IDO, CD40Ig and IL10 in PIECs

Indoleamine 2,3-dioxygenase expression was observed by Western blot analysis of PIEC-IDO cell lysates. A band of the appropriate size $(50 \mathrm{kDa})$ was detected (Fig. 1A). This was absent in the antibody staining control. In order to determine if the enzyme produced was functional, PIEC-IDO lysate was used in a standardized assay [17]. This assay gives a measure of IDO activity by determining the rate of kynurenine production from tryptophan. Results are determined with reference to serial dilutions of kynurenine: PIECIDO cells produced $\sim 50 \mu \mathrm{M}$ kynurenine $/ \mathrm{h} / 1 \times 10^{6}$ cells activity using control cell lysate (PIEC-LZ) as a baseline (data not shown). This rate of IDO activity is greater than can be induced in a range of cell lines by IFN- $\gamma$ exposure [17].

CD40Ig, IL10, and CTLA4Ig single expressing stable cell lines were generated by lentiviral transduction of PIECs. Expression of the transgenes was confirmed by detection of the soluble proteins in the $\mathrm{SN}$ of the cloned cells. A protein band of the correct size ( $\sim 50 \mathrm{kDa})$ from several different PIEC-CD40Ig clones $(\mathrm{A}-\mathrm{H})$ was detected on analysis of $\mathrm{SN}$ on Western blot (Fig. 1B). From this, clone B was selected for subsequent experiments. IL10 expression was confirmed by ELISA, SN from PIEC-IL10

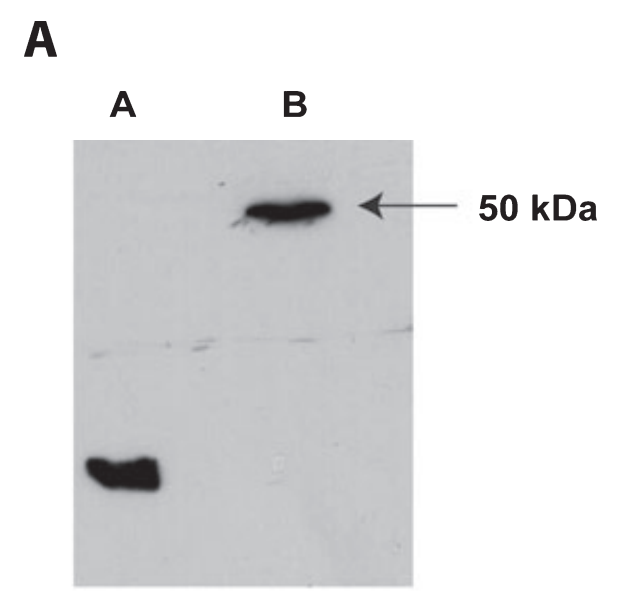

B

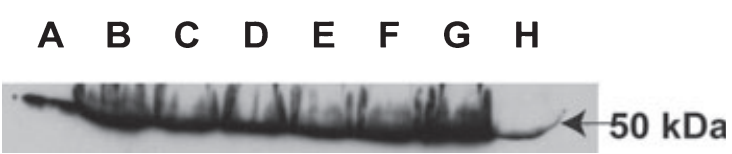

Fig. 1. Expression of CD40Ig, IL10 and IDO by stable PIEC lines. (A) Cell lysate was prepared from PIEC-IDO cells and run on an SDS-PAGE gel. The blot was probed for IDO, with a band of approximately $50 \mathrm{kDa}$ detected. Control cell (PIECLacZ) lysate did not produce a band. The left lane contains an antibody positive control. (B) Tissue culture supernatant from PIEC stable clones transduced with lentivirus-CD40Ig was run on an SDS-PAGE gel and probed with anti-HuIg antibody. All clones A-H produced an appropriately sized band (approximately $50 \mathrm{kDa}$ ) whilst control cells did not. Clone B was selected for use. 
clone (clone 2) expressed $290 \mathrm{ng}$ of IL10 per day $/ 1 \times 10^{6}$ cells (data not shown) was selected for subsequent experiments. The PIEC-CTLA4Ig cell line has been previously described [4].

Local IDO expression inhibits primed xenogeneic proliferative responses in vitro

Proliferation assays used primed BALB/c splenocytes as responders because naïve responders do not proliferate significantly in response to PIECs (our observations and [16]). The effects of single transgene expression by PIECs on primed BALB/c (anti-PIEC) xenogeneic proliferative responses were examined. PIEC-IDO cells inhibited the primed proliferative response by $49 \%, \mathrm{P}=0.02$ compared with control (PIEC-LacZ cells) (Fig. 2A). PIEC-IL10 cells inhibited the primed proliferative response by $37 \%(\mathrm{P}=0.03)$ (Fig. 2B) whilst PIEC-CD40Ig cells increased proliferative responses by $59 \%(\mathrm{P}=0.02)$ (Fig. $2 \mathrm{C}$ ) compared with PIEC-LacZ cells. PIEC-IDO cells consistently were the most potent at reducing primed proliferative responses in these assays and hence were examined further.

Local IDO expression inhibits primed xenogeneic T-cell cytokine responses in vitro

The effects of local IDO expression on cytokine responses (Fig. 3) in the primed xenogeneic assay described above were also assessed. The T-helper 1 (Th1) cytokine IFN- $\gamma$ and the T-helper 2 (Th2) cytokines interleukin-4 (IL4) and IL10 were measured by ELISPOT assay. PIEC-IDO cells inhibited both Th1 and Th2 cytokine responses from responder splenocytes significantly compared to control PIECs. The ELISPOT results presented in Fig. 3A$\mathrm{C}$ show reductions in splenocytes producing IFN- $\gamma$ by $45 \%, \mathrm{P}=0.005$, IL 4 by $57 \%, \mathrm{P}=0.01$ and IL10 by $95 \%, P=0.006$ when exposed to PIECIDO, as compared with control PIECs. Hence, local IDO expression significantly inhibits primed proliferative responses and Th1 and Th2 cytokine responses from splenocytes responding via the indirect antigen presentation pathway.

\section{PIEC-IDO cells co-expressing CD40lg, IL10 or CTLA4lg}

To determine whether further inhibition of xenoresponses could be achieved by the expression of a second transgene with IDO, dual expressing cells were generated by lentiviral transduction of PIECIDO cells. Co-expression was determined by Western blotting of cell lysate for ongoing expression of IDO (Fig. 4A,B) whilst tissue culture supernatant
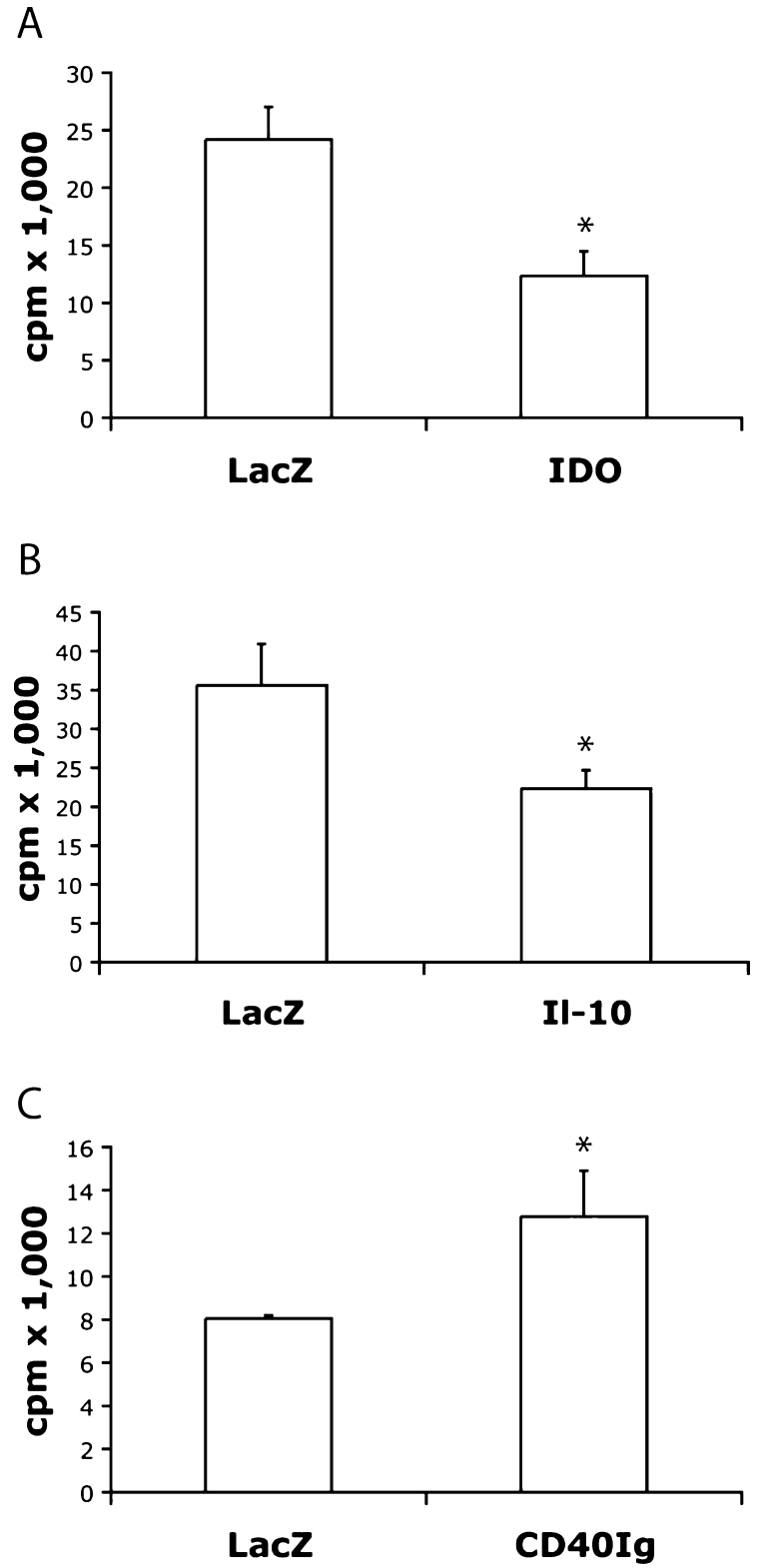

Fig. 2. PIEC-IDO inhibits a primed indirect xenogeneic proliferation to a greater extent than PIEC-IL10 or PIEC-CD40Ig. A primed proliferation assay was performed using $B A L B / c$ splenocytes as responder cells and the stable expressing PIEC cells as stimulator cells. BALB/c mice had been primed by intraperitoneal injection of wild-type PIECs 10 days prior to the assay. The PIECs had been $\gamma$-irradiated. PIECs expressing IDO (panel A) inhibited the proliferation assay by $49 \%$, $\mathrm{P}=0.02$ compared with control (PIEC-LacZ) cells. PIECIL10 cells inhibited proliferation by $37 \%, P=0.03$ compared with PIEC-LacZ whilst CD40Ig expressing cells increased proliferation compared with PIEC-LacZ by $59 \%(\mathrm{P}=0.02)$. $*$ signify a significant difference $(\mathrm{P}<0.05)$.

was assessed for CTLA4Ig and CD40Ig by Western blotting and for IL10 by ELISA (Fig. 4A,C,D). All clones had detectable IDO expression by Western blot of cell lysate. Five PIEC-IDO-CTLA4Ig clones (A-E) were tested for secretion of CTLA4Ig into SN. Clone A had the greatest blot density both for 
A

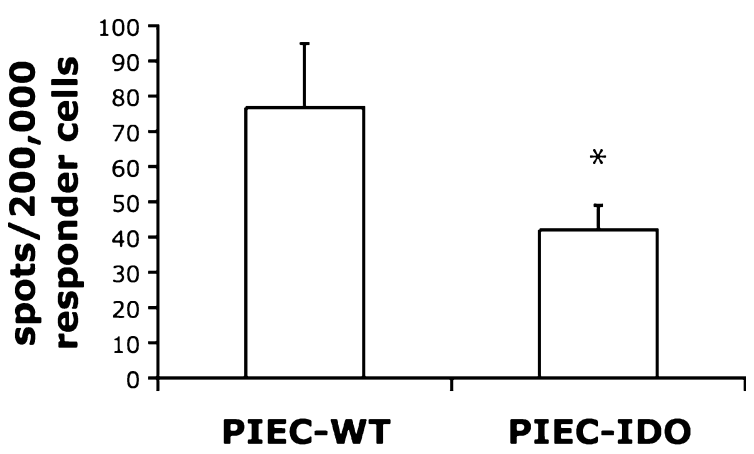

B
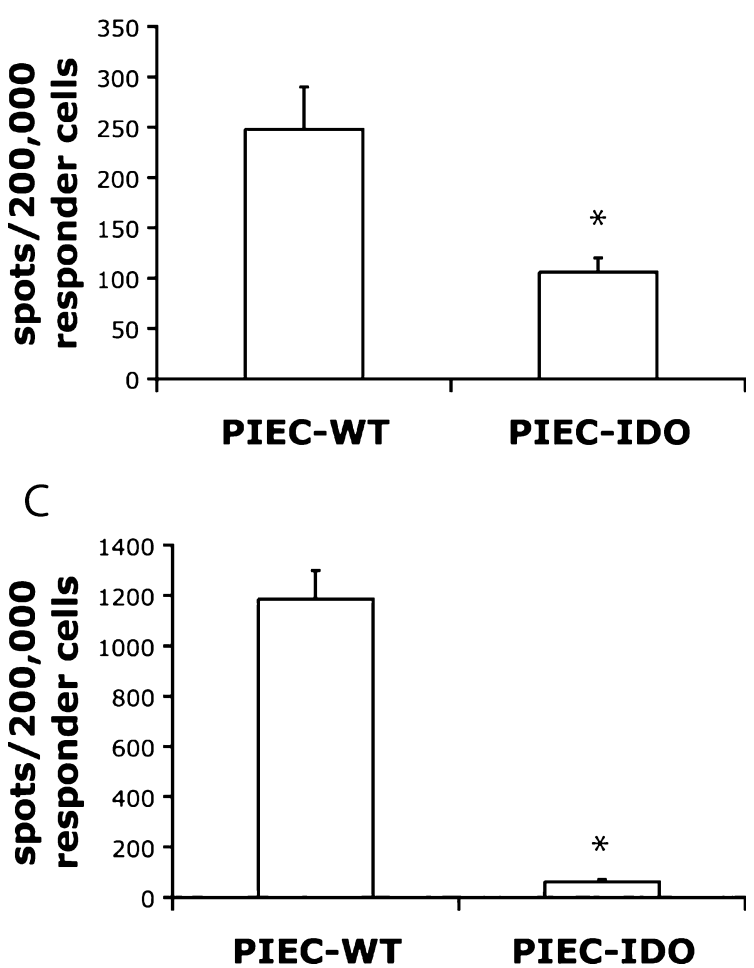

Fig. 3. PIEC-IDO cells reduce primed T helper-1 and T helper2 cytokine production in vitro. ELISPOT assays were performed to assess cytokine production in a primed xenogeneic proliferation assay. Significantly less splenocytes exposed to PIEC-IDO cells produced IFN- $\gamma$ (panel A), IL-4 (panel B) and IL10 (panel C) compared with splenocytes exposed to wild type PIECs (PIEC-WT). * signifies a significant difference compared with control $(\mathrm{P}<0.05)$.

IDO and CTLA4Ig expression and was selected for use (Fig. 4A). Similarly, PIEC-IDO-CD40Ig clone B (Fig. 4C) and PIEC-IDO-IL10 clone 1 (Fig. 4D) were selected for further use because of high coexpression levels.

Dual expressing PIEC-IDO lines inhibit primed proliferative responses in vitro

The dual expressing cell lines were used in the primed proliferation assay as described above.
A

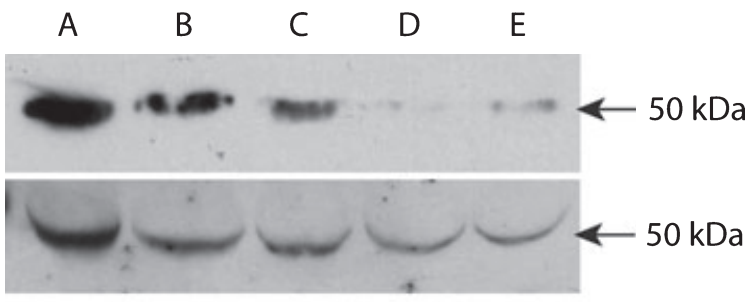

B
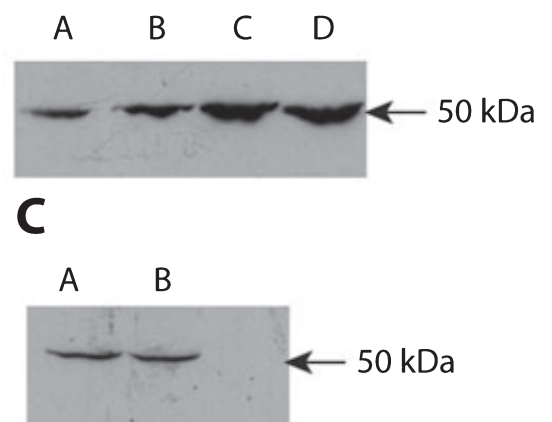

D

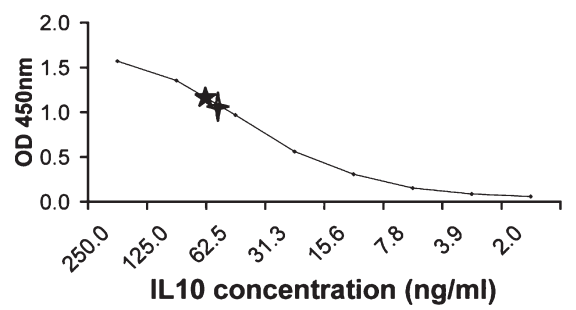

Fig. 4. PIEC-IDO cells co-express CTLA4Ig, CD40Ig, and IL10 after lentiviral transduction. (A) PIEC-IDO-CTLA4Ig clones from PIEC-IDO cells transfected with lentivirusCTLA4Ig and exhibiting resistance to both blasticidin and neomycin (see Materials and methods). The top panel is a Western blot of cell lysate from five clones, probed for IDO whilst the bottom panel is a Western blot of tissue culture supernatant probed with anti-HuIg antibody. All five clones express both transgenes with clone A selected for use due to highest expression of both. (B) Cell lysate from PIEC-IDOCD40Ig clones (A and B) and PIEC-IDO-IL10 clones (C and D) were run on an SDS-PAGE gel and probed for IDO. All clones express IDO. (C) Tissue culture supernatants from PIEC-IDO-CD40Ig clones A and B (from panel B) was run on an SDS-PAGE gel and probed with anti-HuIg. Both clones have a positive band of the appropriate size for CD40Ig (approximately $50 \mathrm{kDa}$ ). Clone $\mathrm{B}$ was selected for use. (D) Tissue culture supernatants from the PIEC-IDO-IL10 clones C and $\mathrm{D}$ described in panel $\mathrm{B}$ were used in an ELISA compared with a standard of known concentration. Clone 1 (five pointed star) was selected for use due to a higher production of IL10 than clone 2 (four pointed star).

PIEC-IDO-CTLA4Ig cells inhibited proliferation by $61 \%(\mathrm{P}=0.04)$ compared with PIEC-IDO and $56 \%(\mathrm{P}=0.03)$ compared with PIEC-CTLA4Ig cells alone (Fig. 5A). This result suggests synergy, 


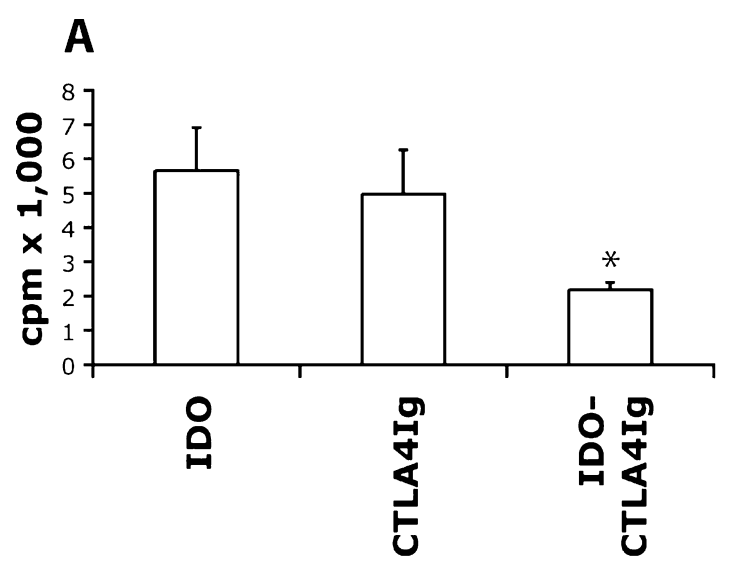

B

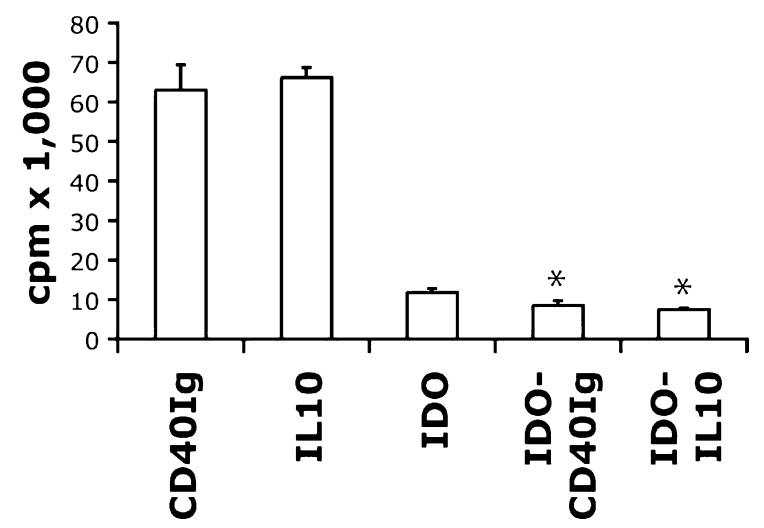

Fig. 5. Co-expression of IDO and CTLA4Ig, CD40Ig or IL10 has additive inhibitory effects on a primed xenogeneic proliferative response. PIECs co-expressing IDO and CTLA4Ig, CD40Ig, and IL10 were applied to primed xenogeneic proliferation assays identical to those described in Fig. 2. Proliferation of primed BALB/c splenocytes in wells containing the co-expressing PIECs was compared to wells containing single expressing cells as controls. (A) PIEC-IDO-CTLA4Ig cells significantly inhibited proliferation in a primed xenogeneic assay compared with both PIEC-IDO and PIEC-CTLA4Ig cells alone. (B) PIEC-IDO-CD40Ig and PIEC-IDO-IL10 cells also inhibited proliferation to a greater extent than PIEC-IDO, PIEC-CD40Ig and PIEC-IL10 cells alone. ${ }^{*}$ signifies a significant difference, compared with PIEC-IDO $\mathrm{P}<0.05$.

given that the PIEC-IDO-CTLA4Ig cells produced less CTLA4Ig per cell than PIEC-CTLA4Ig cells (based on comparative Western blot band densities, data not shown).

The PIEC-IDO-CD40Ig and PIEC-IDO-IL10 cells also inhibited proliferation significantly compared with PIEC-IDO (7\% $\mathrm{P}=0.03$ and $36 \%$, $\mathrm{P}=0.01$ respectively) (Fig. 5B). However IDO, as a single agent, was so efficient at reducing proliferation in these assays that the additional inhibition, with co-expression, appear of limited significance. PIEC-IDO-CD40Ig cells inhibited proliferation by $86 \%, \mathrm{P}=0.004$ compared with PIEC-CD40Ig cells and PIEC-IDO-IL10 cells inhibiting proliferation by $89 \%, \mathrm{P}<0.001$ compared with PIEC-IL10 cells (Fig. 5B).

PIEC-IDO xenografts inhibit lymphocyte infiltration after transplantation under the renal capsule in naïve mice

The in vivo effects of expression of IDO, CD40Ig, IL10, CTLA4Ig and co-expression of IDO with the other molecules on xenograft survival was next examined. PIECs embedded in recipient blood clots were transplanted under the renal capsule of $\mathrm{BALB} / \mathrm{c}$ mice (see Materials and methods). Grafts were recovered at days 7 and 14 for histological assessment. Infiltrating lymphocytes, neutrophils, and surviving PIECs were counted (Table 1A,B). PIEC-GFP grafts were used as controls.

At day 7, PIEC-GFP grafts exhibited intense lymphocytic infiltration with minimal neutrophil infiltrate (Table 1A). PIEC-GFP grafts contained a mean of $61 \pm 4$ lymphocytes per field. Lymphocytic infiltrate was reduced by $27 \% \mathrm{P}<0.001$ in PIEC-IDO grafts and by $39 \% \mathrm{P}<0.001,31 \%$ $\mathrm{P}<0.001$, and $28 \% \mathrm{P}<0.001$ for PIEC-IDOCTLA4Ig, PIEC-IDO-CD40Ig and PIEC-IDOIL10 co-expressing grafts respectively (Table 1A). PIEC-IDO-CTLA4Ig grafts contained the least infiltrate, with a trend toward less infiltrate than PIEC-IDO grafts (Table 1A) and significantly less infiltrate than PIEC-CTLA4Ig grafts, $29 \% \quad \mathrm{P}<0.01$. There was a trend toward reduced infiltration compared with PIEC-IL10 for PIEC-IDO-IL10 and PIEC-CD40Ig for

Table 1. Summary of days 7 and 14 histology findings

\begin{tabular}{llll}
\hline Graft type & Lymphocytes & Neutrophils & Graft cells \\
\hline (A) Day 7 & & & \\
GFP & $61 \pm 3.8$ & $0.8 \pm 0.3$ & $64 \pm 2.5$ \\
CTLA4lg & $53 \pm 2.4^{*}$ & $1.4 \pm 0.4$ & $71 \pm 3.9$ \\
CD40lg & $49 \pm 3.8^{*}$ & $0.9 \pm 0.2$ & $73 \pm 2.6^{*}$ \\
IL10 & $55 \pm 4.2$ & $1.3 \pm 0.4$ & $63 \pm 3.3$ \\
IDO & $45 \pm 2.6^{*}$ & $2.8 \pm 0.7^{*}$ & $84 \pm 2.0$ \\
IDO-CTLA4lg & $37 \pm 3.4^{*}$ & $3.3 \pm 1.1^{*}$ & $74 \pm 4.4$ \\
IDO-CD40lg & $42 \pm 4.7^{*}$ & $6.1 \pm 1.1^{*}$ & $69 \pm 2.4$ \\
IDO-IL10 & $44 \pm 3.3^{*}$ & $5.4 \pm 0.6^{*}$ & $66 \pm 2.6$ \\
(B) Day 14 & & & \\
GFP & $62 \pm 7.0$ & $7.7 \pm 2.2$ & $57 \pm 5.3$ \\
CTLA4lg & $38 \pm 4.6^{*}$ & $4.4 \pm 0.9$ & $58 \pm 3.3$ \\
CD40lg & $19 \pm 3.5^{*}$ & $4.0 \pm 1.0$ & $42 \pm 3.8^{*}$ \\
IL10 & $37 \pm 10^{*}$ & $4.1 \pm 0.9$ & $42 \pm 7.8$ \\
ID0 & $12 \pm 2.8^{*}$ & $0.8 \pm 0.4^{*}$ & $40 \pm 4.3^{*}$ \\
IDO-CTLA4lg & $19 \pm 5.1^{*}$ & $3.6 \pm 1.1$ & $56 \pm 6$ \\
IDO-CD40lg & $17 \pm 4.1^{*}$ & $5.6 \pm 0.7$ & $48 \pm 5.9$ \\
IDO-IL10 & $26 \pm 3.2^{*}$ & $5.0 \pm 1.4$ & $62 \pm 5.2$ \\
\hline
\end{tabular}

Naiive BALB/C mice received PIEC grafts in a recipient blood clot under the left renal capsule. Grafts were harvested at (A) day 7 and (B) day $14(n=3$ per graft type) for histological assessment. Numbers of lymphocytes, neutrophils and graft cells (PIECS) were counted per field at regular intervals along the graft (as described in Materials and methods). Mean \pm standard errors are displayed. Figures marked with * are significantly different $(P<0.05)$ from PIEC-GFP grafts. 


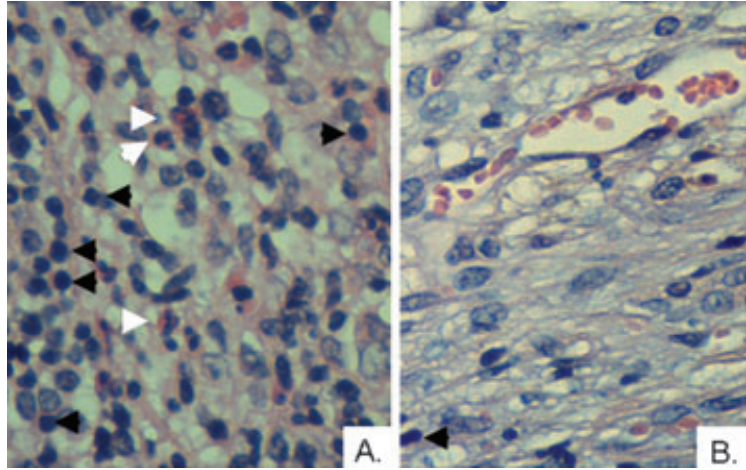

Fig. 6. PIEC-IDO xenografts reduce inflammatory infiltrate at day 14 compared with PIEC-GFP control grafts. PIEC grafts were harvested from BALB/c mice at day 14 . Representative haematoxylin and eosin stained sections from (A) PIEC-GFP and (B) PIEC-IDO grafts. Images are taken at 40× magnification. PIEC-IDO grafts contain significantly less lymphocytic (black arrow heads) and neutrophilic (white arrow heads) infiltrate at this time point. Whilst macroscopically smaller then PIEC-GFP grafts, PIEC-IDO grafts demonstrate a more normal "endothelial" architecture.

PIEC-IDO-CD40Ig. All grafts retained similar numbers of PIECs on day 7 (63 to 84 cells per field). IDO expressing grafts exhibited higher numbers of non-lymphocytic infiltrating cells, compared with controls, which were predominantly neutrophils (Table 1A).

At day 14 PIEC-GFP grafts had the most lymphocytic infiltrate and the single expressing PIEC-IDO grafts the least (Table 1B and Fig. 6). All IDO expressing grafts contained significantly less infiltrate than PIEC-GFP grafts: PIECIDO-IL10 $59 \% \quad(\mathrm{P}<0.001) ; \quad$ PIEC-IDOCTLA4Ig 69\% (P < 0.001); PIEC-IDO-CD40Ig $73 \% \quad(\mathrm{P}<0.001)$; PIEC-IDO $80 \% \quad(\mathrm{P}<0.001)$ (Table 1B).

Despite containing the least infiltrating lymphocytes PIEC-IDO grafts had the least PIECs remaining at day 14 (Table 1B). PIEC-IDO grafts had $27 \%(\mathrm{P}=0.04)$ less PIECs present per field than PIEC-GFP grafts. Despite this PIEC-IDO grafts developed typical endothelial cell morphology with formation of lumens whilst PIEC-GFP grafts demonstrated distorted architecture due to the inflammatory infiltrate (Fig. 6). Neutrophilic infiltrate diminished in PIEC-IDO grafts by day 14. On macroscopic inspection at harvest, PIEC-IDO grafts were considerably smaller than PIEC-GFP grafts, which is likely due to reduced inflammatory cell infiltrate.

\section{Discussion}

Indoleamine 2,3-dioxygenase markedly inhibited primed xenogeneic $\mathrm{T}$-cell proliferation in vitro whilst IL10 and CD40Ig were less effective. Local expression of these immunomodulatory molecules has not been extensively examined and to our knowledge there are no published reports in primed xenogeneic models. IDO is known to have lymphocyte suppressive effects via tryptophan depletion and kynurneine excess, particularly of the catabolites 3-hydroxykunerine, 3-hydroxyanthranilic acid, and quinolate [21-24]. Terness et al. [24] demonstrated that IDO is particularly effective at inducing apoptosis in T-effector cells which is pertinent to this primed model. In addition, Alexander et al. [7] demonstrated inhibition of diabetogenic splenocyte proliferation by IDO expressing islet cells in an allotransplant model.

Consistent with our findings, CD40Ig has not previously been demonstrated to have a role in suppressing primed T-cell responses [25]. Memory T-cell activation is independent of CD40 : CD40L costimulation in allotransplant studies and the present study also indicates that CD40Ig does not inhibit primed indirect xenoresponses in vitro [25]. IL10 appears to have modest anti-proliferative effects on primed xenogeneic splenocytes however as the levels of inhibition were consistently less than those seen with IDO, IDO was examined further. In general IL10 is thought to have proinflammatory effects on primed responses [13,14], however the suppressive effects observed in our model may relate to an increased susceptibility of indirect xenoresponses to IL10 inhibition than when the direct pathway is also available. In addition, combined with IDO expression, IL10 appears to add further suppression. We have previously described the ability of CTLA4Ig local expression to inhibit a primed xenogeneic response in vitro [4].

IDO expression led to potent inhibition of cytokine responses from both primed xenogeneic Th1 and Th2 cells. Fallarino et al. [22] demonstrated that kynurenines tend to inhibit Th1 reponses more than Th2 responses. Tryptophan depletion however, appears to have a pan-suppressive T-cell effect, which may explain why Th2 cytokines are also inhibited in our studies [5]. The current studies examine xenorejection responses via the indirect pathway and this may result in qualitative differences in lymphocyte responses to the previously reported findings in allogeneic settings with both direct and indirect pathways present.

Co-expression of CTLA4Ig, CD40Ig, and IL10 with IDO inhibited in vitro splenocyte proliferation to a greater extent than IDO alone or CTLA4Ig, CD40Ig or IL10 alone. However, the additional suppression from the second molecule 
was not great enough to conclusively state that synergy exists.

There are limited studies describing histology findings in IDO expressing grafts. Alexander et al. [7] transduced islets with adenovirus to achieve IDO overexpression and showed prolonged allograft survival in NOD-SCID mice reconstituted with diabetogenic splenocytes. Histology revealed significant lymphocytic infiltrate around grafts but the authors do not comment on invasion into the graft itself. Li et al. [10] transduced human fibroblasts to express IDO at the site of bovine skin grafts on mice and found significantly reduced infiltration with $\mathrm{CD}^{+}$lymphocytes. Neither study reports early infiltration of grafts with neutrophils such as was seen in the current study, however the use of different time points may account for this discrepancy. Interestingly, a recent report on IDO transfected islets cell clusters in an allotransplant model found increased macrophage infiltration compared with controls despite prolonged survival of IDO expressing grafts (Abstract no. 425, ATC 2007, San Francisco, US). IDO is involved in host defence against pathogens and thus it may be speculated that it also has chemoattractant properties for cells of the innate immune system.

Dual expressing IDO lines were in general less effective than PIEC-IDO cells alone at inhibiting lymphocyte infiltration, albeit significantly more potent than control and PIEC-CTLA4Ig grafts. There have been no previous reported studies examining the effects of co-expression of these molecules on rejection responses. The current in vitro and in vivo findings do not suggest significant benefits of such co-expression within the limitations of the xenorejection experiments conducted. There appear to be some modest benefits from co-expression in terms of reduced proliferative responses in vitro and the PIEC-IDOCTLA4Ig and PIEC-IDO-IL10 grafts exhibited greater numbers of graft cells remaining at day 14 than PIEC-IDO grafts. The PIEC-IDO grafts were noticeably smaller upon macroscopic inspection than other grafts types but the PIEC-IDO cells appeared healthy on microscopic examination. Reduced PIEC-IDO cell numbers in vivo may reflect the high level of IDO expression from these cells inhibiting their own proliferation. We, and others [26] have observed that IDO overexpressing cells after gene transfection have reduced proliferation in vitro. To counteract this effect, developing a transfection system which allows regulation of the level and timing of IDO expression would be valuable.

In conclusion, IDO expression potently suppresses xenorejection responses, activated via the indirect pathway, in vitro and in vivo. Co-expression of IDO with CTLA4Ig and IL10 resulted in added benefits in vitro and in vivo, with respect to graft cell numbers. The present study supports the potential therapeutic role of these immunomodulatory expressed locally in cellular xenografts such as porcine islets for the purpose of facilitating graft acceptance with minimal or no immunosuppression.

\section{Acknowledgments}

This work was supported by funds obtained from the National Health and Medical Research Council of Australia.

\section{References}

1. Auchincloss H JR, Sachs DH. Xenogeneic transplantation. Annu Rev Immunol 1998; 16: 433.

2. Coulombe M, Yang H, Guerder $\mathrm{S}$ et al. Tissue immunogenicity: the role of MHC antigen and the lymphocyte costimulator B7-1. J Immunol 1996; 157: 4790.

3. LeCHLER RI, BAtCHELOR JR. Restoration of immunogenicity to passenger cell-depleted kidney allografts by the addition of donor strain dendritic cells. J Exp Med 1982; 155: 31.

4. Mulley WR, Wee Jl, Christiansen D, Milland J, IERINO FL, SANDRIN MS. Lentiviral expression of CTLA4Ig inhibits primed xenogeneic lymphocyte proliferation and cytokine responses. Xenotransplantation 2006; 13: 248.

5. Mellor AL, Munn DH. IDO expression by dendritic cells: tolerance and tryptophan catabolism. Nat Rev Immunol 2004; 4: 762.

6. Fallarino F, Grohmann U, You $\mathrm{S}$ et al. The combined effects of tryptophan starvation and tryptophan catabolites down-regulate $\mathrm{T}$ cell receptor zeta-chain and induce a regulatory phenotype in naive $\mathrm{T}$ cells. J Immunol 2006; 176: 6752 .

7. Alexander AM, Crawford M, Bertera $\mathrm{S}$ et al. Indoleamine 2,3-dioxygenase expression in transplanted NOD Islets prolongs graft survival after adoptive transfer of diabetogenic splenocytes. Diabetes 2002; 51: 356.

8. Beutelspacher SC, Pillai R, Watson MP et al. Function of indoleamine 2,3-dioxygenase in corneal allograft rejection and prolongation of allograft survival by overexpression. Eur J Immunol 2006; 36: 690.

9. Sun W, Wang Q, Zhang L et al. Blockade of CD40 pathway enhances the induction of immune tolerance by immature dendritic cells genetically modified to express cytotoxic $\mathrm{T}$ lymphocyte antigen 4 immunoglobulin. Transplantation 2003; 76: 1351.

10. Li Y, Tredget EE, Ghaffari A et al. Local expression of indoleamine 2,3-dioxygenase protects engraftment of xenogeneic skin substitute. J Invest Dermatol 2006; 126: 128.

11. Clarkson MR, SAyegh MH. T-cell costimulatory pathways in allograft rejection and tolerance. Transplantation 2005; 80: 555 .

12. Larsen CP, Knechtle SJ, Adams A, Pearson T, Kirk AD. A new look at blockade of T-cell costimulation: a therapeutic strategy for long-term maintenance immunosuppression. Am J Transplant 2006; 6: 876. 


\section{IDO inhibits indirect xenoresponses}

13. Mocellin S, Marincola F, Rossi CR, NitTi D, Lise M. The multifaceted relationship between IL-10 and adaptive immunity: putting together the pieces of a puzzle. Cytokine Growth Factor Rev 2004; 15: 61.

14. Moore KW, de WaAl Malefyt R, Coffman RL, O'GARRA A. Interleukin-10 and the interleukin-10 receptor. Annu Rev Immunol 2001; 19: 683.

15. Bluestone JA, St Clair EW, Turka la. CTla4Ig: bridging the basic immunology with clinical application. Immunity 2006; 24: 233.

16. Yun S, Rose ML, Fabre JW. The induction of major histocompatibility complex class II expression is sufficient for the direct activation of human CD4 $+\mathrm{T}$ cells by porcine vascular endothelial cells. Transplantation 2000; 69: 940.

17. Takikawa O, Kuroiwa T, Yamazaki F, Kido R. Mechanism of interferon-gamma action. Characterization of indoleamine 2,3-dioxygenase in cultured human cells induced by interferon-gamma and evaluation of the enzyme-mediated tryptophan degradation in its anticellular activity. J Biol Chem 1988: 263: 2041.

18. Solomon MF, Ramshaw IA, Simeonovic CJ. Recombinant fowlpox virus for in vitro gene delivery to pancreatic islet tissue. Immunol Cell Biol 2005; 83: 615.

19. Nikfarjam M, Muralidharan V, Christophi C. Altered growth patterns of colorectal liver metastases after thermal ablation. Surgery 2006; 139: 73.
20. Nikfarjam M, Muralidharan V, Su K, MalcontentiWilson C, Christophi C. Patterns of heat shock protein (HSP70) expression and Kupffer cell activity following thermal ablation of liver and colorectal liver metastases. Int J Hyperthermia 2005; 21: 319.

21. Bauer TM, Jiga LP, Chuang JJ et al. Studying the immunosuppressive role of indoleamine 2,3-dioxygenase: tryptophan metabolites suppress rat allogeneic $\mathrm{T}$-cell responses in vitro and in vivo. Transpl Int 2005; 18: 95.

22. Fallarino F, Grohmann U, VacCa $\mathrm{C}$ et al. $\mathrm{T}$ cell apoptosis by tryptophan catabolism. Cell Death Differ 2002; 9: 1069.

23. Frumento G, Rotondo R, Tonetti M et al. Tryptophan-derived catabolites are responsible for inhibition of $\mathrm{T}$ and natural killer cell proliferation induced by indoleamine 2,3-dioxygenase. J Exp Med 2002; 196: 459.

24. Terness P, Bauer TM, Rose L et al. Inhibition of allogeneic $\mathrm{T}$ cell proliferation by indoleamine 2,3-dioxygenaseexpressing dendritic cells: mediation of suppression by tryptophan metabolites. J Exp Med 2002; 196: 447.

25. LONDON CA, Lodge MP, ABbAs AK. Functional responses and costimulator dependence of memory CD4 + T cells. J Immunol 2000; 164: 265.

26. Keskin DB, Marshall B, Munn D, Mellor AL, GEARHART DA. Decreased protein nitration in macrophages that overexpress indoleamine 2, 3-dioxygenase. Cell Mol Biol Lett 2007; 12: 82. 